\title{
Pityriasis rubra pilaris
}

INSERM

\section{Source}

INSERM. (1999). Orphanet: an online rare disease and orphan drug data base. Pityriasis rubra pilaris. ORPHA:2897

Pityriasis rubra pilaris is a rare chronic papulosquamous disorder of unknown etiology characterized by small follicular papules, scaly red-orange patches, and palmoplantar hyperkeratosis, which may progress to plaques or erythroderma. Although most of the cases are sporadic and acquired, a familial form of the disease exists. 